\title{
RECICLAGEM DE PLACAS DE CIRCUITO IMPRESSO DE COMPUTADORES SUCATADOS PARA A RECUPERAÇÃO DE ESTANHO POR ELETROELUIÇÃO DE RESINAS POLIMÉRICAS DE TROCA IÔNICA
}

\footnotetext{
A. H. MARTINS ${ }^{1}$, H. W. A. SILVA ${ }^{1}$, A. E. C. PERES ${ }^{1}$, C. A. PEREIRA ${ }^{2}$, M. L. TOREM ${ }^{3}$ E A. J. B. DUTRA ${ }^{4}$

${ }^{1}$ Departamento de Engenharia Metalúrgica e de Materiais-Universidade Federal de Minas Gerais (UFMG).

${ }^{2}$ Departamento de Engenharia de Minas-Universidade Federal de Ouro Preto (UFOP).

${ }^{3}$ Departamento de Engenharia de Materiais-Pontifícia Universidade Católica do Rio de Janeiro(PUCRJ).

${ }^{4}$ Programa de Engenharia Metalúrgica e de Materiais-Universidade Federal do Rio de Janeiro (UFRJ). afonsohmartins@yahoo.com.brahmartin@demet.ufmg.br
}

Artigo submetido em junho/2013 e aceito em junho/2014

DOI: $10.15628 /$ holos.2014.1513

\section{RESUMO}

Este trabalho apresenta os resultados experimentais para a recuperação de estanho presente em soluções aquosas a parir da reciclagem de computadores sucatados, através da técnica de eletroeluição de resina polimérica catiônica Amberlite IR- $120^{\circledR}$ (Merck, USA). A resina foi carregada pela passagem de $200 \mathrm{ml}$ de solução aquosa $0,2 \mathrm{~g} / \mathrm{l}$ de Sn em 2,18N H2SO4 (concentração do licor de lixiviação de placas de circuito impresso- $\mathrm{PCl}$ de microcomputadores sucatados) através do leito de $10 \mathrm{~g}$ de resina. A realização dos experimentos foi baseada em um planejamento estatístico pelo método fatorial completo replicado de três variáveis a dois níveis. A otimização foi feita pelo método do passo ascendente. As condições consideradas ótimas visando a eletrorrecuperação de estanho foram 150A/m2 de densidade de corrente catódica, tempo de eletroeluição de 45 minutos e intensidade de agitação moderada do eletrólito (100rpm).

PALAVRAS-CHAVE: estanho, reciclagem, sucata eletrônica, eletroeluição, hidrometalurgia.

\section{RECYCLING OF PRINTED CIRCUIT BOARDS FROM SCRAPPED COMPUTERS FOR TIN RECOVERY BY ELECTROELUTION OF ION EXCHANGE POLYMERIC RESIN}

\begin{abstract}
This work presents experimental results for tin recovery from synthetic aqueous solution based on leaching liquors for computers recycling using the electroelution technique of cationic polymeric resin Amberlite IR-120 ${ }^{\circledR}$ (Merck, USA). The resin was loaded with $200 \mathrm{ml}$ of aqueous solution $0.2 \mathrm{~g} / \mathrm{I} \mathrm{Sn}$ in $2.18 \mathrm{~N} \mathrm{H} 2 \mathrm{SO} 4$ (same metal content of a leaching liquor of printed circuit board-PCl from scrapped computers) through $10 \mathrm{~g}$ of polymeric
\end{abstract}

resin bed. The experiments were statistically planned by a replicated full factorial design method for three variables at two levels. The optimization was performed by the ascending path statistical method. The optimum experimental conditions for maximum tin recovery were $150 \mathrm{~A} / \mathrm{m} 2$ for current density, 45 minutes for electroelution and fair stirring intensity around 100rpm.

KEYWORDS: tin, recycling, electronic scrap, electroelution, hydrometallurgy. 


\section{INTRODUÇÃO}

Os equipamentos eletrônicos fazem parte do cotidiano do homem na sociedade moderna. As inovações tecnológicas não só ampliam as aplicações e usos desses equipamentos, mas também fazem com que esses equipamentos tornem-se obsoletos muito rapidamente. O volume de equipamento eletrônico usado, em especial os microcomputadores pessoais, continua aumentando em todo o planeta. Segundo dados do Greenpeace, são produzidos até 50 milhões de toneladas/ano desse tipo de dejeto no mundo inteiro e o volume vem crescendo em $5 \%$ ao ano na Europa (Greenpeace, 2013).

No Brasil, quem procura descartar um microcomputador obsoleto deve procurar uma empresa de reciclagem, todavia, poucas são as empresas que atuam nesse ramo de atividade com tecnologia apropriada e condições de preservação ambiental. Assim, a sucata eletrônica no Brasil, muitas das vezes, acaba sendo despejada nos aterros sanitários junto com o lixo doméstico. Para evitar a disposição nos aterros industriais, esses microcomputadores usados deveriam ser coletados e processados para reutilização parcial ou total de seus componentes e/ou para a recuperação de metais, vidros, plásticos e materiais em geral.

Um breve levantamento do estado da arte de reciclagem de computadores usados (Castro e Martins, 2009) mostra que as rotas tecnológicas para a recuperação de metais, a partir de sucatas eletrônicas, têm se caracterizado pela geração de emissões que são potencialmente tão perigosas quanto os metais sob tratamento, especialmente, os metais pesados presentes nas estruturas dessas sucatas. Isso demonstra a necessidade de agregação de valor tecnológico inovador a essas rotas de tratamento metalúrgico, principalmente, para a indústria brasileira de reciclagem de sucatas eletrônicas.

Entre as tecnologias alternativas para recuperação de metais, a partir das suas soluções aquosas, pode ser mencionada a de troca iônica com resinas poliméricas (Fleming e Cromberge, 1984). As principais vantagens do uso de resinas poliméricas nos sistemas de recuperação de metais são: elevadas taxas de adsorção metálica, etapas de regeneração da resina com custo financeiro-operacional inferior a outras técnicas hidrometalúrgicas, estabilidade química nas condições de processo, inexpressiva perda de resina ao longo dos processos, com um baixo custo de reposição, além da possibilidade de reciclagem e regeneração da resina permitindo sua reutilização. Além dessas características, deve-se ressaltar o aspecto de baixa contaminação do meio ambiente em decorrência da sua utilização.

A eletroeluição de resinas poliméricas para a recuperação de metais (Zdorova, 1967; Bek, 1972; Martins, 1991) consiste na remoção dos íons metálicos adsorvidos a resina, utilizando o efeito combinado do deslocamento físico e/ou reação química com outros íons presentes no eletrólito. Estes efeitos são estimulados pelo campo elétrico gerado na célula de eletroeluição, o qual fornece a força motriz necessária ao sistema para estimular a migração destas espécies iônicas até a superfície do catodo para ocorrer a deposição metálica.

Este trabalho experimental apresenta os resultados da eletroeluição de resinas poliméricas catiônicas Amberlite IR- $120^{\circledR}$ (Merck,USA), associada à eletrorrecuperação de estanho a partir de um eletrólito aquoso sintético com composição química similar ao do licor de lixiviação com $\mathrm{H}_{2} \mathrm{SO}_{4}$ 
da rota hidroeletrometalúrgica adotada para o tratamento de placas de circuito impresso $(\mathrm{PCl})$ de microcomputadores usados destinados ao sucateamento.

\section{METODOLOGIA}

As placas de circuito impresso (PCI) de microcomputadores sucatados utilizadas nos experimentos foram fornecidas pelo Setor de Manutenção de Microcomputadores da Escola de Engenharia da Universidade Federal de Minas Gerais.

As placas de circuito impresso foram processadas segundo o procedimento descrito por Martins (2007). As PCl foram desmontadas visando remover as partes não-metálicas e os componentes eletrônicos considerados indesejáveis. A maioria das partes metálicas encontradas nas placas de circuito impresso estava fixada por soldagem ou prensagem. As placas desmontadas foram cortadas em guilhotina manual e os fragmentos obtidos foram alimentados em um pulverizador elétrico de panela (ou moinho de panela), marca PAVITEST, modelo I-4227 com temporizador. A moagem foi efetuada por 8 minutos e o produto foi peneirado utilizando-se uma peneira com abertura de malhas padrão de $0,208 \mathrm{~mm}$ (65mesh da série Tyler). 0 material retido na peneira foi realimentado no moinho de panela para remoagem.

O produto da etapa de moagem e com granulometria abaixo de $0,208 \mathrm{~mm}$ foi lavado com água destilada em béqueres de vidro borossilicato com capacidade nominal de $2000 \mathrm{ml}$. A lavagem foi efetuada em três etapas com $1000 \mathrm{ml}$ de água destilada em cada uma. O objetivo da etapa de lavagem foi a remoção da parte considerada não metálica do material moído que poderia interferir potencialmente na etapa posterior de lixiviação.

Cobre $(2,85 \%)$, estanho $(3,09 \%)$ e alumínio $(3,10 \%)$ foram as espécies de maior concentração no pó lavado e foram determinadas por espectrofotometria de absorção atômica (AA). Este trabalho concentrou-se na recuperação de Sn. Todas as determinações das concentrações de $\mathrm{Sn}$ em soluções aquosas produzidas neste trabalho também foram efetuadas por espectrometria de absorção atômica(AA) (Perkin Elmer, mod. AAnalyst300-USA).

A resina catiônica Amberlite IR- $120^{\circledR}$ foi, inicialmente, mergulhada em um banho de água destilada por 24 horas visando promover seu "inchamento", isto é, absorção de água na sua estrutura. Em seguida, a resina foi filtrada e colocada em bandejas retangulares para secagem ao ar. Após a secagem, a resina foi alimentada a um becher contendo solução $2,18 \mathrm{~N} \mathrm{H}_{2} \mathrm{SO}_{4}$ para o condicionamento químico da resina por 24 horas. O carregamento metálico da resina catiônica Amberlite IR- $120^{\circledR}$ foi efetuado em uma coluna vertical de troca iônica por percolação descendente da solução aquosa portadora de estanho. A coluna vertical consistiu de uma bureta graduada (capacidade nominal de $50 \mathrm{ml}$ ) com $620 \mathrm{~mm}$ de comprimento e $10 \mathrm{~mm}$ de diâmetro interno. Fez-se a adição de, aproximadamente, $40 \mathrm{ml}$ de água destilada para preencher a coluna vertical de troca iônica. Certa quantidade apropriada de lã de vidro foi posicionada na parte inferior e interior da coluna para servir como suporte do leito de resina polimérica a ser formado durante o carregamento metálico. A massa de $10 \mathrm{~g}$ de resina IR-120 ${ }^{\circledR}$ condicionada e seca foi pesada em balança analítica. Essa massa foi transferida para a coluna vertical de troca iônica preenchida com água destilada. A vazão de passagem de solução aquosa através da coluna vertical de troca iônica foi regulada para $10 \mathrm{ml} . \mathrm{min}^{-1}$. O carregamento do leito de resina formado ocorreu através da passagem de $200 \mathrm{ml}$ de solução aquosa $0,2 \mathrm{~g} / \mathrm{l}$ de $\mathrm{Sn}$ em $2,18 \mathrm{~N} \mathrm{H}_{2} \mathrm{SO}_{4}$, através do leito de $10 \mathrm{~g}$ de 
resina. Após a passagem de $200 \mathrm{ml}$ de solução aquosa, o procedimento foi repetido três vezes com a mesma solução aquosa inicialmente empregada no carregamento.

Ao final de cada procedimento, recolheu-se a solução passante pelo leito de resina e armazenou-se em um frasco de vidro para análise química visando determinar a concentração final de estanho.

Uma vez concluído o carregamento metálico do leito de resina polimérica, a massa de resina foi removida da coluna vertical e deixada secar ao ar por 24 horas. A resina seca foi armazenada em sacos plásticos para ser utilizada nos experimentos de eletroeluição.

Os experimentos de eletroeluição foram realizados a temperatura ambiente e consistiram no emprego de uma célula eletrolítica de seção quadrada $(26 \mathrm{~mm}$ de lado e $50 \mathrm{~mm}$ de altura) de vidro borosilicato com capacidade nominal de $30 \mathrm{ml}$ cuja representação esquemática é mostrada na figura 1 e inspirada no trabalho de Martins (1993).

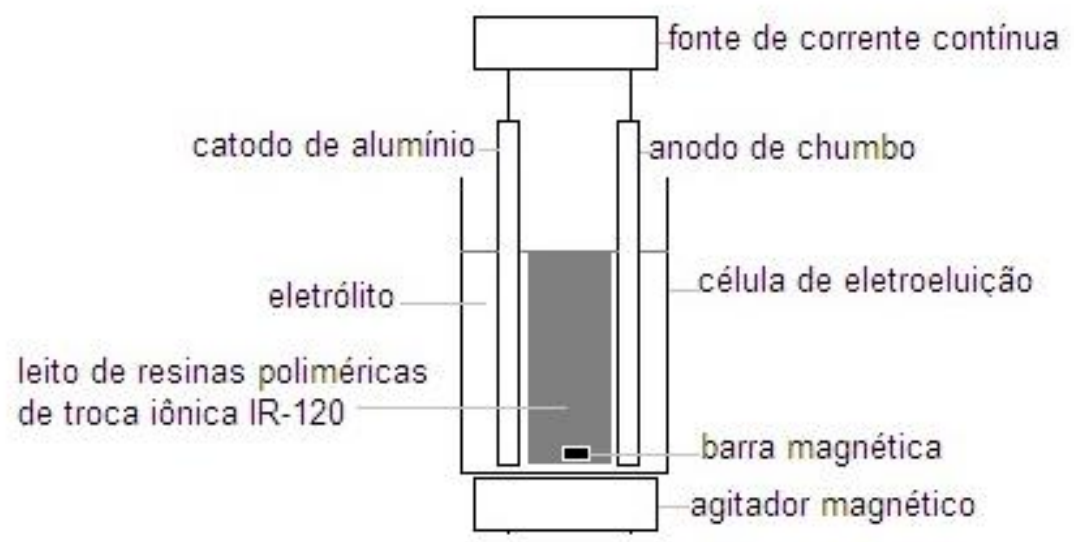

Figura 1- Representação esquemática de célula de eletroeluição empregada nos experimentos e inspirada no trabalho de Martins (1993).

O eletrólito da célula de eletroeluição foi formado por $10 \mathrm{~g}$ de resina seca, condicionada e carregada, imersos em $15 \mathrm{ml}$ de solução aquosa $2,18 \mathrm{~N} \mathrm{H}_{2} \mathrm{SO}_{4}$. Os eletrodos foram imersos no eletrólito. Uma fonte elétrica (Minipa, mod.MPL-1303M) de corrente contínua foi conectada aos terminais de um catodo de alumínio e um anodo de chumbo metálico, ambos com 70mm de altura, $20 \mathrm{~mm}$ de largura e $0,2 \mathrm{~mm}$ de espessura. As espessuras do catodo e anodo foram desconsideradas no cálculo das densidades de corrente.

A densidade de corrente empregada nos experimentos foi determinada com base na área do catodo de alumínio imerso no eletrólito, isto é, a parte do catodo que toma parte das reações de redução das espécies metálicas iônicas na região do católito. O valor dessa área foi $6,4 \times 10^{-4} \mathrm{~m}^{2}$.

Um multímetro potenciométrico (Agilent, mod. U3401A) foi empregado nos experimentos e permitiu acompanhar os valores de voltagem e corrente catódicas contínuas aplicadas na célula de eletroeluição. Uma barra magnética com imã ( $5 \mathrm{~mm}$ de comprimento) foi imersa no eletrólito aquoso e seu acionamento deu-se por uma placa aquecedora com agitação magnética (Fisatom, mod. 752A), a qual promoveu a agitação do eletrólito naqueles experimentos em que a agitação 
foi adotada. Naqueles experimentos em que houve a agitação do eletrólito/leito de resina polimérica, a intensidade de agitação foi da ordem de 100rpm, aproximadamente, e considerada moderada.

As correntes foram calculadas com base na área dos eletrodos submersa na solução, a área submersa foi de $6,4 \times 10^{-4} \mathrm{~m}^{2}$ para cada eletrodo, sabendo-se que a densidade de corrente é a razão entre a corrente elétrica catódica por unidade de área do catodo imerso no eletrólito.

Ao final do experimento, desligaram-se a fonte de corrente contínua e a placa aquecedora com agitação magnética e transferiram-se os eletrodos da célula para um vidro de relógio, deixando-os secar ao ar por 24 horas. Os eletrodos foram pesados e a massa depositada no catodo foi calculada por diferença entre a massa final e a massa inicial de eletrodo. A massa inicial do catodo foi medida antes do experimento em uma balança analítica de precisão(Top Loading, série mark S).

No final de cada experimento, o eletrólito usado e a resina utilizada foram recolhidas para análise química visando a determinação da concentração metálica presente.

Nos experimentos de eletroeluição de resina polimérica para a recuperação de estanho, as massas metálicas depositadas sobre o catodo foram determinadas por pesagem direta do catodo em balança analítica, antes e após cada experimento. A pesagem dos catodos em balança analítica foi precedida de lavagem com água destilada e aspersão de acetona líquida (PA) visando acelerar a evaporação do excesso de água na superfície do catodo de alumínio.

A realização dos experimentos foi baseada em um planejamento estatístico pelo método fatorial completo replicado de três variáveis a dois níveis (Box et. al. ,1978; Cox, 1958). As variáveis adotadas neste trabalho foram a densidade de corrente catódica, o tempo de eletroeluição e a intensidade de agitação do eletrólito. Para cada uma das variáveis foram adotados dois níveis experimentais, um superior e outro inferior. Os valores dos níveis experimentais para cada variável foram determinados com base em experimentos preliminares exploratórios. Oito experimentos replicados, com um total de 16, foram executados. Foram utilizados 16 catodos de alumínio, um para cada experimento.

A tabela 1 mostra as variáveis e os níveis experimentais adotados.

Tabela 1 - Variáveis adotadas e seus respectivos níveis experimentais

\begin{tabular}{|l|l|l|l|}
\hline VARIÁVEIS & NOTAÇÃO & \multicolumn{2}{|l|}{ NÍVEIS EXPERIMENTAIS } \\
\cline { 3 - 4 } & & SUPERIOR(+) & INFERIOR(-) \\
\hline Densidade de corrente catódica $\left(\mathrm{A} / \mathrm{m}^{2}\right)$ & A & $\mathbf{2 0 0}$ & $\mathbf{1 0 0}$ \\
\hline Tempo de eletroluição (min) & B & $\mathbf{3 0}$ & $\mathbf{2 0}$ \\
\hline Intensidade de agitação do eletrólito $(\mathrm{min})$ & $\mathbf{C}$ & com agitação (100rpm) & sem agitação \\
\hline
\end{tabular}

$\mathbf{R}_{1}, \mathbf{R}_{\mathbf{2}}$-resposta experimental replicada expressa em massa metálica(g) depositada no catodo

\section{RESULTADOS}

A análise por difratometria de raios- $X$ do pó gerado pela moagem das placas desmontadas de circuitos impressos de microcomputadores sucatados, e lavado com água destilada, mostrou a presença de $\mathrm{Sn}$ metálico e de $\mathrm{SnO}_{2}$. 
O teor de Sn encontrado foi igual a 3,09\% e é comparável a maioria das fontes minerais primárias (Sevryukov et al., 1977), razão pela qual o Sn foi selecionado para estudo.

A escolha da concentração $2,18 \mathrm{~N} \mathrm{H}_{2} \mathrm{SO}_{4}$ objetivou avaliar o comportamento de solubilização de estanho presente no pó de alimentação, uma vez que a reciclagem de metais encoraja o uso de reagentes de baixo custo e em menores concentrações relativas visando elevar o retorno financeiro do projeto e, também, reduzir eventuais impactos ambientais.

A lixiviação com solução $2,18 \mathrm{~N} \mathrm{H}_{2} \mathrm{SO}_{4}$ e borbulhamento de oxigênio foi efetuada com $50 \mathrm{~g}$ de pó lavado, oriundo do processamento das $\mathrm{PCl}$ de microcomputadores sucatados. A massa de pó foi lixiviada com $500 \mathrm{ml}$ de solução $2,18 \mathrm{~N} \mathrm{H}_{2} \mathrm{SO}_{4}$ em um béquer de vidro borossilicato de $1000 \mathrm{ml}$ de capacidade nominal. A polpa formada foi mantida a $60 \pm 2^{\circ} \mathrm{C}$ e sob intensa agitação magnética por 120 minutos, empregando uma placa aquecedora com agitação magnética. Amostras do licor de lixiviação foram coletadas, filtradas, armazenadas em frascos de amostra de vidro borossilicato, identificadas e encaminhadas para determinação da concentração de Sn em solução através de espectrometria de absorção atômica (AA). Os resultados experimentais indicaram que, aproximadamente, 2,0g/l de Sn foram extraídos correspondendo a 64,7\% do Sn inicialmente presente no pó processado de $\mathrm{PCl}$.

A eficiência de carregamento metálico do leito de resina polimérica foi determinada pela razão entre a massa adsorvida na resina após a passagem da solução aquosa e a massa inicialmente presente na solução aquosa de carregamento.

A tabela 2 mostra os resultados obtidos para os experimentos replicados de eletroeluição de resina polimérica catiônica IR-120 ${ }^{\circledast}$ para a eletrorrecuperação de estanho. Os resultados foram expressos em massa de estanho (gramas) eletrodepositado sobre o catodo.

Tabela 2 - Respostas experimentais obtidas a partir da Matriz de blocos do método fatorial completo para experimentos replicados de eletroeluição de resinas poliméricas IR-120 ${ }^{\circledast}$ para a eletrorrecuperação de estanho.

\begin{tabular}{|c|c|c|c|c|c|c|}
\hline EXPERIMENTO & A & B & C & NOTAÇÃO & \multicolumn{2}{|c|}{ Sn(g) } \\
\cline { 5 - 7 } & & & & & $R_{1}$ & $R_{2}$ \\
\hline 1 & - & - & - & T & $\mathbf{0 , 0 0 5}$ & $\mathbf{0 , 0 0 5}$ \\
\hline 2 & + & - & - & A & $\mathbf{0 , 0 0 5}$ & $\mathbf{0 , 0 1 5}$ \\
\hline 3 & - & + & - & B & $\mathbf{0 , 0 2 0}$ & $\mathbf{0 , 0 5 0}$ \\
\hline 4 & + & + & - & AB & $\mathbf{0 , 0 4 0}$ & $\mathbf{0 , 0 4 0}$ \\
\hline 5 & - & - & + & C & $\mathbf{0 , 0 1 0}$ & $\mathbf{0 , 0 1 0}$ \\
\hline 6 & + & - & + & AC & $\mathbf{0 , 0 1 0}$ & $\mathbf{0 , 0 1 0}$ \\
\hline 7 & - & + & + & BC & $\mathbf{0 , 0 4 0}$ & $\mathbf{0 , 0 4 0}$ \\
\hline 8 & + & + & + & ABC & $\mathbf{0 , 0 5 0}$ & $\mathbf{0 , 0 5 0}$ \\
\hline
\end{tabular}

Amostras de solução aquosa após o carregamento da resina IR-120 ${ }^{\circledR}$ foram encaminhadas para análise química via espectrometria de absorção atômica(AA). Levando-se em consideração a concentração inicial da solução aquosa de carregamento antes do contato com o leito de resinas poliméricas, se chegou ao resultado de 0,02g de $\mathrm{Sn} / \mathrm{g}$ de resina seca, ou seja, 1,0g de resina seca continha $0,02 \mathrm{~g}$ de estanho.

Os resultados experimentais obtidos para a eletroeluição da resina IR-120 ${ }^{\circledR}$ visando a recuperação de estanho mostrados na tabela 2 possibilitaram a construção do algoritmo de Yates do método fatorial completo a dois níveis experimentais e mostrado na tabela 3. 
Em razão dos resultados obtidos pela construção do algoritmo de Yates, verificou-se que a variável tempo de eletroeluição foi a única considerada significativa ao nível de $95 \%$ de confiança estatística (ou 5\% de erro estatístico), para os níveis experimentais estudados neste trabalho para cada variável analisada.

Tabela 3 - Algoritmo de Yates construído a partir dos resultados para a eletroeluição da resina IR-120 ${ }^{\circledR}$ visando a recuperação de estanho

\begin{tabular}{|c|c|c|c|c|c|c|c|c|c|}
\hline EXP. & R1+R2 & $\mathrm{Y}-1$ & $\mathrm{Y}-2$ & $\mathrm{Y}-3$ & $\mathrm{DM}$ & EFEITO & $\left(\mathrm{R}_{1}-\mathrm{R}_{2}\right)^{2}$ & Tcalc & Significância \\
\hline 1 & 0,01 & 0,03 & 0,18 & 0,40 & 0,05 & $\mathbf{T}$ & 0,0001 & - & - \\
\hline 2 & 0,02 & 0,15 & 0,22 & 0,04 & 0,005 & A & 0,0004 & 1,07 & NS \\
\hline 3 & 0,07 & 0,04 & 0,02 & 0,26 & 0,0325 & B & 0,0001 & 6,95 & S \\
\hline 4 & 0,08 & 0,18 & 0,02 & 0,02 & 0,0025 & AB & 0,0000 & 0,53 & NS \\
\hline 5 & 0,02 & 0,01 & 0,12 & 0,04 & 0,005 & C & 0,0000 & 1,07 & NS \\
\hline 6 & 0,02 & 0,01 & 0,14 & 0,00 & 0,000 & AC & 0,0000 & 0 & NS \\
\hline 7 & 0,08 & 0,00 & 0,00 & 0,02 & 0,0025 & BC & 0,0000 & 0,535 & NS \\
\hline 8 & 0,10 & 0,02 & 0,02 & 0,02 & 0,0025 & ABC & 0,0000 & 0,535 & NS \\
\hline
\end{tabular}

A partir dos valores da coluna DM da tabela 3, pode-se verificar que a variável tempo de eletroeluição (B) apresentou a maior influência positiva sobre a resposta experimental (massa de estanho recuperada). Isto é, maiores tempos de eletroeluição correspondem a maiores massas de estanho recuperadas. Desta forma, mais espécies metálicas podem ser reduzidas na superfície do catodo e ocorrer a eletrodeposição metálica. As outras variáveis, densidade de corrente $(A)$ e intensidade de agitação do eletrólito(C) e as interações ( $A B, A C, B C$ e $A B C$ ) não foram significativas estatisticamente ao nível de $95 \%$ de confiança (ou $5 \%$ de erro).

O efeito positivo da variável tempo de eletroeluição sobre a eletrorrecuperação de estanho era esperado. O tempo é parâmetro fundamental para permitir que os íons estanho desorvidos da superfície das esferas de resina IR-120 ${ }^{\circledR}$, devido ao efeito do campo elétrico gerado na célula de eletroeluição, possam migrar através do eletrólito e alcançar a superfície do catodo onde ocorrem as reações de redução e formação do depósito metálico de estanho.

A otimização dos resultados experimentais obtidos através do método de otimização pelo passo ascendente foi realizada. A tabela 4 apresenta as respostas estatisticamente previstas pelo método de otimização pelo passo ascendente. Considerando-se que foram utilizadas $10 \mathrm{~g}$ de resina seca $(0,02 \mathrm{~g}$ de estanho para cada grama de resina seca) nos experimentos de eletroeluição, os experimentos de otimização projetados apresentados na tabela 4 foram limitados até o número 6 porque não se pode recuperar uma massa maior do que $0,20 \mathrm{~g}$ de estanho contidos em $10 \mathrm{~g}$ de resina.

Portanto, as condições consideradas estatisticamente ótimas para a eletroeluição da resina polimérica catiônica $\mathrm{IR}-120^{\circledR}$ visando a recuperação de estanho são: densidade de corrente catódica igual a $150 \mathrm{~A} / \mathrm{m}^{2}$, tempo de eletroeluição igual a 45 minutos e com agitação moderada do eletrólito (100rpm, aproximadamente). 
Tabela 4 - Resposta prevista para a recuperação de estanho pelo método do passo ascendente em função dos passos estabelecidos para as variáveis estudadas.

\begin{tabular}{|c|c|c|c|c|}
\hline $\begin{array}{c}\text { EXPERIMENTO } \\
\text { PROJETADO }\end{array}$ & A & B & C & $\begin{array}{c}\text { RESPOSTA PREVISTA } \\
\text { Sn(g) }\end{array}$ \\
\hline 1 & $\mathbf{1 5 0}$ & $\mathbf{1 0}$ & com agitação & $\mathbf{0 , 0 2}$ \\
\hline 2 & $\mathbf{1 5 0}$ & $\mathbf{1 7}$ & com agitação & $\mathbf{0 , 0 6}$ \\
\hline 3 & $\mathbf{1 5 0}$ & $\mathbf{2 4}$ & com agitação & $\mathbf{0 , 0 9}$ \\
\hline 4 & $\mathbf{1 5 0}$ & $\mathbf{3 1}$ & com agitação & $\mathbf{0 , 1 3}$ \\
\hline 5 & $\mathbf{1 5 0}$ & $\mathbf{3 8}$ & com agitação & $\mathbf{0 , 1 6}$ \\
\hline 6 & $\mathbf{1 5 0}$ & $\mathbf{4 5}$ & com agitação & $\mathbf{0 , 2 0}$ \\
\hline
\end{tabular}

Um experimento replicado com as condições ótimas estatisticamente calculadas foi realizado e encontrou-se um valor médio igual a 0,190g de estanho. Nessas condições otimizadas, 95\% do estanho inicialmente adsorvidos na resina polimérica catiônica foram recuperados.

\section{CONCLUSÕES}

O processamento metalúrgico das placas de circuito impresso de microcomputadores sucatados mostrou-se factível ao empregar-se a rota hidroeletrometalúrgica proposta neste trabalho experimental ao nível de bancada de laboratório. A desmontagem das $\mathrm{PCl}$, seguida de corte em pequenos pedaços do suporte das $\mathrm{PCl}$, moagem e lavagem do pó com água destilada permitiu a obtenção de um pó processado com a maior parte dos metais associados.

A lixiviação do pó processado com solução aquosa $2,18 \mathrm{~N}$ de $\mathrm{H}_{2} \mathrm{SO}_{4}$ e borbulhamento de oxigênio possibilitou a solubilização daquelas espécies metálicas de maior valor agregado com vistas à reciclagem, isto é, $2,0 \mathrm{~g} / \mathrm{l}$ de estanho.

O carregamento da resina polimérica catiônica IR- $120^{\circledR}$ deu-se através do emprego de coluna vertical de troca iônica cujo resultado permitiu um carregamento de $0,02 \mathrm{~g}$ de $\mathrm{Sn} / \mathrm{g}$ de resina seca para os experimentos de recuperação individual de estanho.

A realização dos experimentos de eletroeluição da resina $I R-120^{\circledR}$ utilizando o método fatorial completo das três variáveis estudadas permitiu concluir que a única variável significativa, ao nível de $95 \%$ de confiança estatística, para a eletrorrecuperação de estanho, foi o tempo de eletroeluição. Os valores considerados ótimos para a eletrorrecuperação de estanho determinados pelo método estatístico de otimização do passo ascendente foram $150 \mathrm{~A} / \mathrm{m}^{2}$ para a densidade de corrente catódica, 45 minutos de eletroeluição e intensidade de agitação moderada de 100rpm.

De um modo geral, os resultados experimentais obtidos para a recuperação de estanho pela eletroeluição da resina polimérica catiônica IR-120 ${ }^{\circledR}$ a partir da reciclagem de $\mathrm{PCls}$ de computadores destinados ao sucateamento podem ser considerados satisfatórios. Isto porque o valor de $95 \%$ de estanho recuperado (a partir do estanho presente no pó lavado de $\mathrm{PCl}$ processado) pela técnica de eletroeluição de resinas poliméricas pode ser comparado com o resultado de 38,3\% para a recuperação de estanho, obtido em circunstâncias semelhantes, pela técnica de precipitação de sais inorgânicos de estanho para a reciclagem de placas de circuito impresso de computadores sucatados (Martins, 2007). Este valor de 95\% de recuperação de estanho demonstra o potencial da técnica. Não obstante esse potencial, a técnica de eletroeluição de resinas 
poliméricas ainda carece de um maior desenvolvimento tecnológico para permitir seu emprego mais amplo na recuperação de outros metais.

\section{AGRADECIMENTOS}

Os autores agradecem ao Conselho Nacional de Desenvolvimento Científico e Tecnológico (CNPq) e a Fundação de Amparo à Pesquisa do Estado de Minas Gerais (FAPEMIG) pelo apoio financeiro a este trabalho.

\section{REFERÊNCIAS BIBLIOGRÁFICAS}

1. Bek, R. Y. Possibility Using the Electrolytic Elution Process for Ion-Exchange Extraction of Gold and Silver, Tsvet. Met., pp.82-83, 1972.

2. Box, G. E. P., Hunter, W.G, Hunter, J. S. Statistics for Experiments, John Wiley \& Sons Inc., 653p., 1978.

3. Castro, L. A., Martins, A. H. Recovery of tin and copper by recycling of printed circuit boards from obsolete computers. Brazilian Journal of Chemical Engineering, v. 26, n. 04, pp. 649-657, October-December, 2009.

4. Cox, D. R. Planning of Experiments, John Wiley \& Sons Inc., New York, 208 p., 1958.

5. Fleming, C.A., Cromberge, G. The Extraction of Gold from Cyanide Solutions by Strong - and Weak-base Anion Exchange Resins, J. S. Afr. Inst. Min. Metall., v. 84, n.5, May, pp. 125-137, 1984.

6. Fridman, I. D. Regeneration of an AP2 Anion Exchanger in the Ion Exchange of Gold, Soviet Journal of Non-Ferrous Metals, 12, pp. 70-74, 1971.

7. http://www.greenpeace.org/international/en/campaigns/toxics/electronics/the-e-wasteproblem/ acessado em 04/06/2013.

8. Martins, A. H. Recuperação de Ouro e Prata por Electrostripping de Resinas Poliméricas, 460 Congresso Anual da ABM, São Paulo, v. 2, Setembro, pp. 519-540, 1991.

9. Martins, A.H. The Extraction of Gold and Silver by Electroelution of Strong-base Polymeric Resins. Canadian Metallurgical Quartertly, v.32, n.1, January, pp.85-87, 1993.

10. Martins, Afonso Henriques. Reciclagem de Placas de Circuito Eletrônico de Microcomputadores Usados para a Recuperação de Metais. Relatório Técnico de final de atividades de Auxílio no âmbito do Edital CNPq no 019/2004 - Processo 475146/2004-2, Julho 2007, 76 p.

11. Sevryukov, N., Kuzmin, B., Chelishchev, Y., General Metallurgy, Peace Publishers, Moscow, URSS, 1977, $545 \mathrm{p}$.

12. Zdorova, E. P. Desorption of Gold During Electroeluction, Tr. Tsent. Nauch. Iss. Gor. Inst., v.70, pp.58-61, 1967.

13. Wan, R. Y., Miller, J. D. Solvation Extraction and Electrodeposition of Gold from Cyanide Solutions, Journal of Metals, December, pp.35-40, 1986. 